\title{
Relationship among the HDLc, NonHDLc/ HDLc ratio and Gilbert's Syndrome
}

\author{
Salim Ozenc ${ }^{1 *}$, Hakan Sarlak², Sinan Iscen ${ }^{3}$
}

\begin{abstract}
Objective: We investigated the relationship between HDL-cholesterol levels that is indirect marker of ApoA-I production in liver, Non-HDL-cholesterol that is indirect marker of ApoB and GS

Methods: HDLc, NonHDLc/HDLc Ratio levels were investigated in subjects with GS ( $\mathrm{n}=148)$ and compared to healthy controls $(n=148)$.

Results: Age and BMI distributions were similar between the two groups. HDLc levels were lower in GS than the healthy controls $(\mathrm{p}=0.012)$. However, TC, Non-HDLc, Non-HDLc/HDLc levels were higher in GS than the healthy controls $(\mathrm{p}=0.002, \mathrm{p}=0.001, \mathrm{p}<0.001$, respectively). In correlation analysis, UB were negatively mildly correlated with HDLc $(\mathrm{r}=-0.191, \mathrm{p}=0.001)$ and positively correlated with $\mathrm{TC}(\mathrm{r}=0.436, \mathrm{p}<0.001)$, Non-HDLc $(\mathrm{r}=0.511, \mathrm{p}<0.001)$ and Non-HDLc/HDLc $(\mathrm{r}=0.512, \mathrm{p}<0.001)$ in the whole group.

Conclusions: The contrary of previously studies, we would like to suggest that bilirubin is a only antioxidant agent that protects from cardiovascular disease, but not physiological hypolipidemic agent
\end{abstract}

Key words: Gilbert's syndrome (GS), Unconjugated hyperbilirubinemia (UB), HDL cholesterol, Non-HDLcholesterol, NonHDLc/HDLc ratio.

\section{Introduction}

Gilbert's syndrome (GS) is characterized by intermittent unconjugated hyperbilirubinemia in the absence of haemolysis or underlying liver disease. It is a relatively common condition in the general population (3-17\%), depending on the ethnicity studies (1). In most subjects, the hyperbilirubinemia of GS manifests itself during adolescence or early adulthood. The total serum bilirubin concentration usually rises and fluctuates between 20 and $50 \mu \mathrm{mol} / 1$ but rarely exceeds $85 \mu \mathrm{mol} / \mathrm{l}$ (2). Gilbert syndrome is the result of a defect in the promotor of the gene that encodes the enzyme uridine diphosphoglucuronateglucuronosyltransferase 1A1 (UGT1A1), which is responsible for the conjugation of bilirubin with glucuronic acid. A number of studies have reported that gilbert syndrome is negatively associated with the prevalence of cardiovascular disease (CVD) $(3,4)$. However, the mechanisms of decreased frequency of atherosclerotic disease in GS are not entirely known but probably multifactorial.

ApoA-I is produced by the liver and acquires free cholesterol and phospholipid from liver and peripheral cells to form high density lipoprotein
(HDL) cholesterol. An inverse relationship between the level of HDL cholesterol and the presence or development of coronary heart disease (CHD) is well established (5).

Non-HDL-cholesterol, which is estimated by subtracting HDLc from total cholesterol, corresponds closely to measurements of ApoB (6). With respect to the NonHDLc/ HDLc ratio, the UK Prospective Diabetes Study found NonHDLc/HDLc ratio to be better than NonHDLc as a predictor of coronary heart disease (CHD) in patients with type 2 diabetes (7). A recent observational study also demonstrated that the NonHDLc/HDLc ratio is a better marker than the apoB/apoA1 ratio for identifying metabolic syndrome and insulin resistance (8). In the present study, we investigated the relationship between HDL-cholesterol levels that is indirect marker of ApoA-I production in liver, Non-HDL-cholesterol that is indirect marker of ApoB and GS. In order to prevent any interference of confounding factors for inflammation or atherosclerosis, we studied a specifically selected group having no additional disorders such as hypertension, diabetes mellitus or obesity 
Materials and Methods

\section{Subjects}

We recruited a total of one hundred forty eight male patients with GS from the outpatient clinic of the department of Cardiology and Internal Medicine, Diyarbakır Military Hospital. Age, sex and body mass index (BMI) matched one hundred forty eight male healthy volunteers were studied as control group. The diagnosis of GS was made by unconjugated hyperbilirubinemia (UB> $1 \mathrm{mg} / \mathrm{dl}$ ). Eligibility criteria were as follows (3); no evidence of haemolysis (normal full blood count and lactate dehydrogenase $[\mathrm{LDH}]$ ), normal liver enzyme test results (aspartate aminotransferase [AST], alanine aminotransferase [ALT] and male sex. Subjects were excluded if they had a history of liver disease, diabetes mellitus, renal disease, alcoholism, cholelithiasis, coronary heart disease, haemolysis, haemoglobinopathy, positive hepatitis B surface antigen (HBsAg) or anti-hepatitis $\mathrm{C}$ virus (HCV) test, or had used any drugs in the past two weeks. All patients with GS were asymptomatic. The study was approved by the local ethics committee of Gulhane School of Medicine and all participants signed informed consent.

Samples

All blood samples were collected from an antecubital vein, using Vacutainer ${ }^{\circledR}$ tubes (collected in clot activator and EDTA separately) between 08:00 and 09:00 AM after an overnight fasting. All serum and plasma samples were protected from light.

Laboratory investigations

Total and direct bilirubin levels were measured by Olympus AU400 auto-analyser using reagents from Olympus Diagnostics (Hamburg, Germany). UB was calculated by the Formula (UB=total bilirubin-conjugated bilirubin). Also, glucose, total cholesterol (TC), triglyceride (TG), high-density lipoprotein cholesterol (HDLc), lowdensity lipoprotein cholesterol (LDL-C), LDH, AST, ALT and ALT levels were measured by the enzymatic methods with Olympus AU400 auto-analyser using reagents from Olympus Diagnostics (Hamburg, Germany). LDL-C was calculated by Friedewald's formula (9).

\section{Statistical analysis}

Results are reported as the mean \pm SD. Kolmogorov Smirnov test was used to determine the distribution characteristics of variables. Differences between groups were tested for significance by independent samples $\mathrm{t}$ test. To determine the relationship between GS and HDLc variable, multivariate linear regression models were used. The relationship between variables was analysed by Pearson correlation. Differences were considered significant at $\mathrm{p}<0.05$. All data were analysed using SPSS 22.0 (SPSS Inc., Chicago, IL, USA).
Results

The characteristics of the patients and the controls are summarized in Table 1. Age and BMI distributions were similar between the two groups. FBG, LDL-C, TG, AST, ALT levels were also similar in two groups. TC, HDLc, Non-HDLc, NonHDLc/HDLc levels were different. HDLc levels were lower in GS than the healthy controls $(p=0.012)$ (Table 1). However, TC, Non-HDLc, NonHDLc/HDLc levels were higher in GS than the healthy controls $(p=0.002, \quad p=0.001, \quad p<0.001$, respectively) (Table 1). In correlation analysis, UB were negatively mildly correlated with HDLc $(\mathrm{r}=-0.191, \mathrm{p}=0.001)$ and positively correlated with TC $(\mathrm{r}=0.436, \mathrm{p}<0.001)$, Non-HDLc $(\mathrm{r}=0.511, \mathrm{p}<0.001)$ and Non-HDLc/HDLc $(\mathrm{r}=0.512, \mathrm{p}<0.001)$ in the whole group (Figure 1). On the other hand in subgroup analysis no significant association were found between UB and other parameters investigated. In multivariate linear regression analysis taking account HDLc as a dependent variable, HDLc were associated with UB (Beta:-0.181,t:19.322, $\mathrm{p}=0.002$ ) .

\section{Discussion and Conclusion}

To the contrary of our knowledge, the present study shows for the first time TC $(\mathrm{r}=0.436, \mathrm{p}<0.001)$, non-HDLc $(\mathrm{r}=0.511, \mathrm{p}<0.001)$ and non-HDLc/HDLc $(\mathrm{r}=0.512, \mathrm{p}<0.001)$, well known mediators of initial stages of atherosclerosis, was higher in subjects with GS when compared to healthy controls.

These novel findings must be carefully evaluated in the pathogenesis of atherogenesis in GS. Because of several studies have reported an inverse relationship between the presences of CAD and circulating bilirubin levels $(10,11)$. In 1994, Schwertner et al. were the first to observe a significant inverse correlation between bilirubin plasma concentrations and the prevalence of CAD. This important finding indicated that a lower than normal serum bilirubin concentration is associated with the presence of ischemic heart disease (10). Moreover, Vitek et al. recently showed that GS subjects have low prevalence of CAD and presumed chronic hyperbilirubinemia prevent the development of CAD by increasing the serum antioxidant capacity (3). But these findings suggest that antioxidant capacity of serum bilirubin.

In aspect of liver, ApoA-I is produced by the liver and acquires free cholesterol and phospholipid from liver and peripheral cells via the ATP-binding cassette transporter A1 (ABCA1) to form nascent (discoidal) HDLc particles. But HDLc levels were lower in GS than the healthy controls in our study ( $\mathrm{p}=0.012$ ). HDL-cholesterol levels is indirect marker of ApoA-I production in liver. Therefore, production of nonlipidated apoA-I may be decreased by UB in the liver and mature HDLc particles could be reduced 
Table 1. Basic characteristics of the study population.

Age (years)
Creatinine (mg/dL)
Fasting Glucose (mg/dL)
ALT (IU/L)
AST (IU/L)
UB (mg/dL)
TC (mg/dL)
Triglyceride (mg/dL)
HDL (mg/dL)
LDL (mg/dL)
NonHDL (mg/dL)
NonHDL/HDL
Hgb (g/dL)
Hct (\%)
RBC (K/uL)
PIt (K/uL)
MCV (fL)
RDW (\%)
WBC (K/uL)
Lymphocyte (K/uL)
Neutrophil (K/uL)

\section{Gilbert (n=148)}

$29.9 \pm 12.9$

$0.88 \pm 0.27$

$87.8 \pm 12.7$

$25.5 \pm 15.8$

$27.5 \pm 10.3$

$1.49 \pm 0.47$

$175.0 \pm 44.7$

$112.7 \pm 83.6$

$44.9 \pm 13.9$

$107.6 \pm 34.4$

$130.1 \pm 41.7$

$3.2 \pm 1.4$

$15.1 \pm 1.6$

$43.5 \pm 6.3$

$5.3 \pm 0.6$

$243 \pm 57$

$84.5 \pm 6.8$

$12.6 \pm 1.3$

$6.5 \pm 2.1$

$2.1 \pm 1.2$

$3.8 \pm 1.7$

$\begin{array}{cc}\text { Control }(\mathbf{n}=\mathbf{1 4 8}) & \text { P value } \\ 36.3 \pm 14.7 & .073 \\ 0.78 \pm 0.17 & .051 \\ 89.3 \pm 12.6 & .950 \\ 21.7 \pm 12.1 & .051 \\ 24.9 \pm 8.3 & .077 \\ 0.41 \pm 0.18 & <.001 \\ 116.2 \pm 33.1 & .002 \\ 131.9 \pm 74.3 & .564 \\ 50.7 \pm 17.7 & .012 \\ 115.9 \pm 34.2 & .776 \\ 65.5 \pm 27.6 & .001 \\ 1.4 \pm 0.7 & <.001 \\ 14.3 \pm 6.7 & .193 \\ 41.8 \pm 5.1 & .547 \\ 4.9 \pm 0.6 & .394 \\ 284 \pm 66 & .057 \\ 86.9 \pm 4.9 & .051 \\ 12.9 \pm 1.0 & .134 \\ 7.2 \pm 2.0 & .238 \\ 2.8 \pm 0.8 & .026 \\ 3.9 \pm 1.5 & .083\end{array}$

Abbreviations: UB- unconjugated bilirubin; TC- total cholesterol; HDL- high-density lipoprotein cholesterol; LDL - low-density lipoprotein cholesterol; Hgb- hemoglobin; Hct- hematocrit; RBC- red blood cell; Pltplatelets; MCV- mean corpuscular volume; RDW- red cell distribution width; WBC - white blood cell. Independent samples $t$ test. Values are given as mean \pm standard deviation.
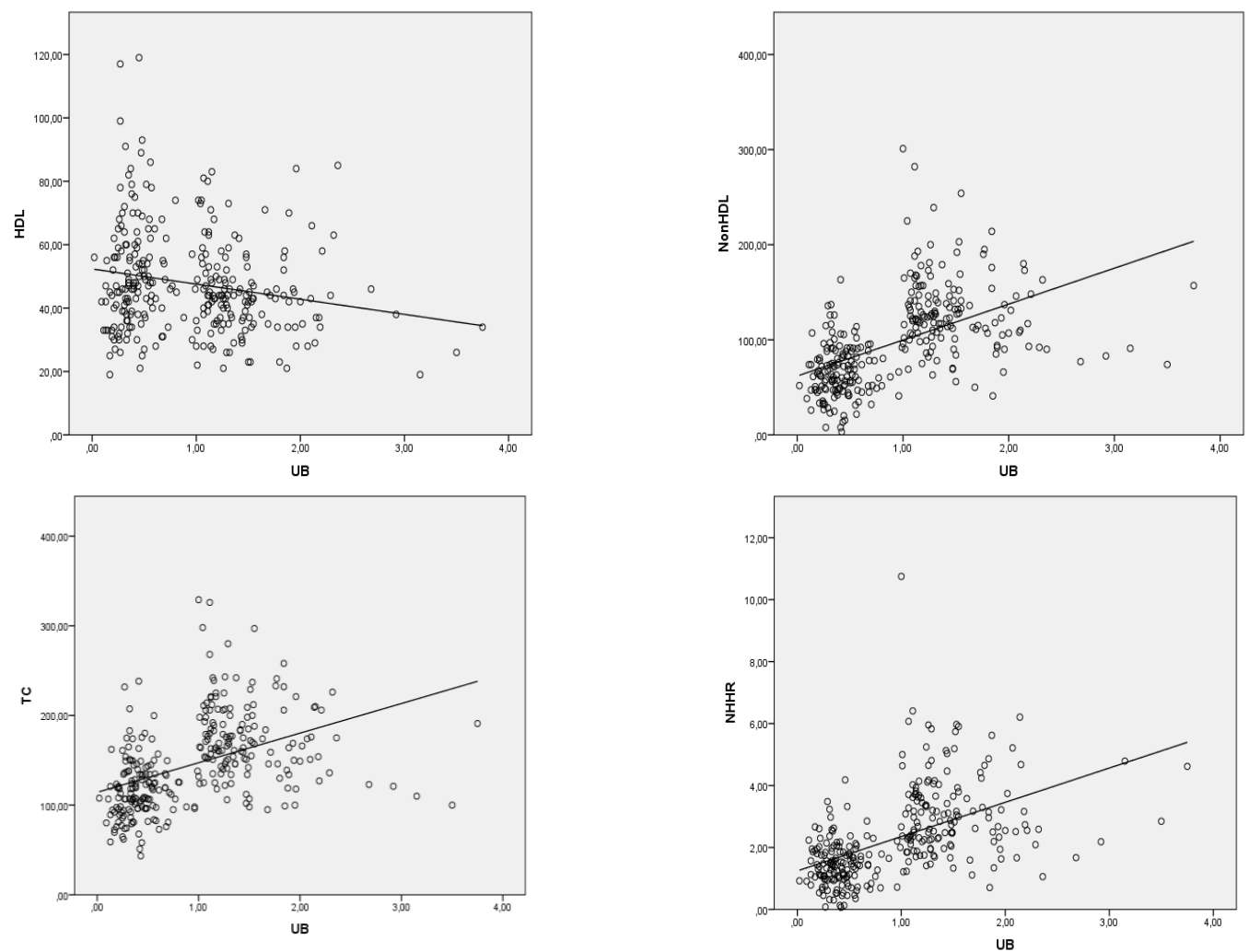

Figure 1. The Pearson correlation analysis in whole group. UB were negatively mildly correlated with HDLc $(\mathrm{r}=-0.191, \mathrm{p}=0.001)$ and positively correlated with $\mathrm{TC}(\mathrm{r}=0.436, \mathrm{p}<0.001)$, non-HDLc $(\mathrm{r}=0.511$, $\mathrm{p}<0.001)$ and non-HDLc/HDLc $(\mathrm{r}=0.512, \mathrm{p}<0.001)$ in the whole group. 
There is no data on the association between the NonHDLc/HDLc ratio and the GS, and also findings that about total lipid profile are controversial (12-15). The NonHDLc/HDLc ratio captures atherogenic lipid abnormalities other than LDLc including abnormalities in lipid particles such as small dense LDL, very low-density lipoproteins (VLDL), and HDLc similarly to that of ApoB/A1 (8). In our study, non-HDLc level as a marker of ApoB levels were found higher in the GS than the healthy controls. These findings may suggest that non-HDL cholesterol esters are taken up by endocytosis into hepatocytes is defective in the GS.

In this study, we found that GS was significantly associated with an increased risk of TC, non-HDLc and non-HDLc/HDLc. At first glance, these findings could appear unexpected. Because several studies have reported an inverse relationship between the presence of CAD and circulating bilirubin levels $(10,11,16)$. But the contrary of these studies $(10,11,16)$, we would like to suggest that bilirubin is a only antioxidant agent that protects from cardiovascular disease, but not physiological hypolipidemic agent

\section{Conclusion}

The contrary of previously studies, we would like to suggest that bilirubin is a only antioxidant agent that protects from cardiovascular disease, but not physiological hypolipidemic agent. Our study has several limitations. Because of the narrow selection criteria, the sample size was small. Hence, our data may not be representative for all subjects with GS.

Conflict of Interest: The authors declare no potential conflicts of interest with respect to the research, authorship, and/or publication of this article.

Acknowledgements: This study was designed and analysed by the authors. The database was collected from the Diyarbakır Military Hospital registry by the authors.

\section{References}

1. Fertrin KY, Goncalves MS, Saad ST, Costa FF. Frequencies of UDP-glucuronosyltransferase 1 (UGT1A1) gene promoter polymorphisms among distinct ethnic groups from Brazil. Am J Med Genet 2002;108:117-9.

2. Hirschfield GM, Alexander GJ. Gilbert's syndrome: an overview for clinical biochemists. Ann Clin Biochem 2006; $43: 340-3$
Vitek L, Jirsa M, Brodanova M, Kalab M, Marecek Z, Danzig V, et al. Gilbert syndrome and ischemic heart disease: a protective effect of elevated bilirubin levels. Atherosclerosis 2002;160:449-56

Schwertner HA. Bilirubin concentration, UGT1A1*28 polymorphism, and coronary artery disease. Clin Chem 2003;49:1039-40.

5. Gordon DJ, Rifkind BM. High-density lipoprotein--the clinical implications of recent studies. N Engl J Med 1989; 321:1311.

6. Lamprea-Montealegre JA, Sharrett AR, Matsushita K, Selvin E, Szklo M, Astor BC. Chronic kidney disease, lipids and apolipoproteins, and coronary heart disease: the ARIC Study. Atherosclerosis 2014;234:42e6.

7. Holman RR, Coleman RL, Shine BS, Stevens RJ. NonHDL cholesterol is less informative than the total-to-HDL cholesterol ratio in predicting cardiovascular risk in type 2 diabetes. Diabetes Care 2005;28:1796e7.

8. Kim SW, Jee JH, Kim HJ, Jin S, Suh S, Bae JC, et al. NonHDL-cholesterol/HDL-cholesterol is a better predictor of metabolic syndrome and insulin resistance than apolipoprotein B/apolipoprotein A1. Int $\mathrm{J}$ Cardiol 2013;168:2678e83.

9. Friedewald WT, Levy R, Fredrickson DS. Estimation of the concentration of low density lipoprotein cholesterol in plasma, without use of the preparative ultracentrifuge. Clin Chem 1972;18:499-502.

10. Schwertner HA, Jackson WG, Tolan G. Association of low serum concentration of bilirubin with increased risk of coronary artery disease. Clin Chem 1994;40:18-23.

11. Hopkins PN, Wu LL, Hunt SC, James BC, Vincent GM, William RR. Higher serum bilirubin is associated with decreased risk for early familial coronary artery disease. Arterioscler Thromb Vasc Biol 1996;16:250-5.

12. Očadlík I, Hlinštáková S, Oravec S. Relationship between unconjugated hyperbilirubinemia and lipoprotein spectrum. Neuro Endocrinol Lett. 2011;32(3):360-4

13. Bulmer AC, Verkade HJ, Wagner KH. Prog Lipid Res. 2013 Apr;52(2):193-205. doi: 10.1016/j.plipres.2012.11.001. Epub 2012 Nov 29.Bilirubin and beyond: a review of lipid status in Gilbert's syndrome and its relevance to cardiovascular disease protection.

14. Effects of Gilbert's Syndrome on Lipid Profile, Levels of Serum Uric Acid, Glucose and Insulin Resistance. Cüre M.C , Cüre E. , Kırbaș A, Yüce S. , Ertürk A. Cukurova Medical Journal 2014;39(3):443-450. 
15. Tapan S, Karadurmus N, Dogru T, Ercin CN, Tasci I, Bilgi $\mathrm{C}$, et al. Decreased small dense LDL levels in Gilbert's syndrome. Clin Biochem 2011;44:300-3.
16. Bilirubin and beyond: A review of lipid status in Gilbert's syndrome and its relevance to cardiovascular disease protection. A.C. Bulmer, H.J. Verkade, K.-H. Wagner. Prog Lipid Res. 2013 Apr;52(2):193-205. doi: 10.1016/j.plipres.2012.11.001. Epub 2012 Nov 29.

Copyright (C) 2014 The Author(s); This is an open-access article distributed under the terms of the Creative Commons Attribution License (http://creativecommons.org/licenses/by/4.0), which permits unrestricted use, distribution, and reproduction in any medium, provided the original work is properly cited. All Rights reserved by international journal of Medical Science and Discovery. 